\title{
Future Pharmacotherapy for Non-alcoholic Steatohepatitis (NASH): Review of Phase 2 and 3 Trials
}

\author{
James J. Connolly ${ }^{1}$, Kohtaro Ooka ${ }^{2}$ and Joseph K. Lim*3 \\ ${ }^{1}$ Department of Internal Medicine, Yale University School of Medicine, New Haven, CT, USA; ${ }^{2}$ Division of Gastroenterology, \\ Hepatology and Nutrition, University of Pittsburgh Medical Center, Pittsburgh, PA, USA; ${ }^{3}$ Yale Liver Center, Section of Digestive
} Diseases, Yale University School of Medicine, New Haven, CT, USA

\begin{abstract}
Non-alcoholic steatohepatitis (NASH) results from inflammation and hepatocyte injury in the setting of hepatic steatosis. Nonalcoholic steatohepatitis increases the risk of progression to liver fibrosis and cirrhosis, and is the most rapidly growing etiology for liver failure and indication for liver transplantation in the USA. Weight loss and lifestyle modification remain the standard first-line treatment, as no USA Food and Drug Administration-approved pharmacotherapy currently exists. The past decade has seen an explosion of interest in drug development targeting pathologic pathways in non-alcoholic steatohepatitis, with numerous phase 2 and 3 trials currently in progress. Here, we concisely review the major targets and mechanisms of action by class, summarize results from completed pivotal phase 2 studies, and provide a detailed outline of key active studies with trial data for drugs in development, including obeticholic acid, elafibranor, cenicriviroc and selonsertib. Citation of this article: Connolly JJ, Ooka K, Lim JK. Future pharmacotherapy for non-alcoholic steatohepatitis (NASH): Review of phase 2 and 3 trials. J Clin Transl Hepatol 2018;6(3):264-275. doi: 10.14218/JCTH.2017.00056.
\end{abstract}

\section{Introduction}

Non-alcoholic steatohepatitis (NASH), a subcategory of nonalcoholic fatty liver disease (NAFLD), is defined as the presence of hepatic steatosis and inflammation with hepatocyte injury. ${ }^{1}$ Approximately one-quarter of the world's population has NAFLD, with estimates of the prevalence of NASH ranging from $1.5 \%$ to $6.45 \% .^{2}$ In the USA, this translates into an estimated $\$ 103$ billion annual economic burden. ${ }^{3}$

NASH is currently the second leading etiology of cirrhosis among adults awaiting liver transplantation in the USA, ${ }^{4}$ and is expected to soon represent the leading indication for liver

Keywords: Fatty liver; Non-alcoholic steatohepatitis; Clinical trials; Pharmacotherapy; Obeticholic acid.

Abbreviations: CCR, C-C motif chemokine receptor; CVC, cenicriviroc; FDA, USA Food and Drug Administration; FGF, fibroblast growth factor; FXR, farnesoid X nuclear receptor; GLP, glucagon-like peptide; HDL, high-density lipoprotein; LDL, low-density lipoprotein; MRI-PDFF, magnetic resonance imaging proton density fat fraction; NAFLD non-alcoholic fatty liver disease; NAS, NAFLD activity score; NASH, non-alcoholic steatohepatitis; NCT, national clinical trial number; OCA, obeticholic acid; PBC, primary biliary cholangitis; PPAR, peroxisome proliferator-activated receptor.

Received: 18 August 2017; Revised: 16 February 2018; Accepted: 4 April 2018

*Correspondence to: Joseph K. Lim, Yale Liver Center, Section of Digestive Diseases, Yale University School of Medicine, 333 Cedar Street, LMP 1080, New Haven, CT 06520-8019, USA. Tel: +1-203-737-6063, Fax: +1-203-785-7273, E-mail: joseph.lim@yale.edu transplantation. ${ }^{5}$ There are currently no USA Food and Drug Administration (FDA) approved medications for the treatment of NASH. Current management is primarily focused on promoting weight loss through lifestyle interventions. Weight loss medications, bariatric surgery and bariatric endoscopy represent attractive future approaches for $\mathrm{NASH}$, although there is limited prospective data to support their role in clinical practice, and they are not presently endorsed by the American Association for the Study of Liver Diseases. ${ }^{1}$

In the last decade, the number of clinical trials of pharmacotherapies for the treatment of NASH has significantly increased. Several systematic reviews and meta-analyses have been conducted to aggregate data from published studies. ${ }^{6-8}$ Therapies investigated with questionable benefit have included metformin, thiazolidinediones, vitamin E and pentoxifylline. However, the heterogeneity of study design and inclusion criteria, modest cohort sizes, differences in reported outcomes (e.g., histologic characteristics), lack of fibrosis improvement, and uncertain long-term benefits and safety have limited interpretation of their therapeutic safety and efficacy. In this context, a growing cohort of clinical development programs evaluating novel pharmacotherapeutic agents for NASH has emerged, primarily focused on demonstrating improvement in histologic characteristics of $\mathrm{NASH}$, including steatosis, steatohepatitis and fibrosis. The aim of this paper is to briefly summarize the targets for these future pharmacotherapies, with a focus on agents in phase 2 and 3 trials.

\section{Methods}

We searched ClinicalTrials.gov in May 2017 for phase 2 or 3 interventional studies that were open for enrollment or active but not enrolling and contained the phrase "non-alcoholic steatohepatitis". Pediatric studies were not included. We did not include trials for nutritional supplements. When there were discrepancies between ClinicalTrials.gov and published materials (e.g., conference abstract), we used the information from the published materials.

\section{Key targets and mechanisms of action}

Drugs in trials for NASH are designed to attenuate lipotoxicity, whether by reducing lipid accumulation or by reducing downstream pathways that lead to hepatocyte injury, death and, in some, cirrhosis. Many drugs in phase 3 trials and those with results from phase 2 trials target lipid metabolism, inflammation or the formation of fibrous connective tissue within the liver (Fig. 1). Examples of agents that target lipid metabolism 


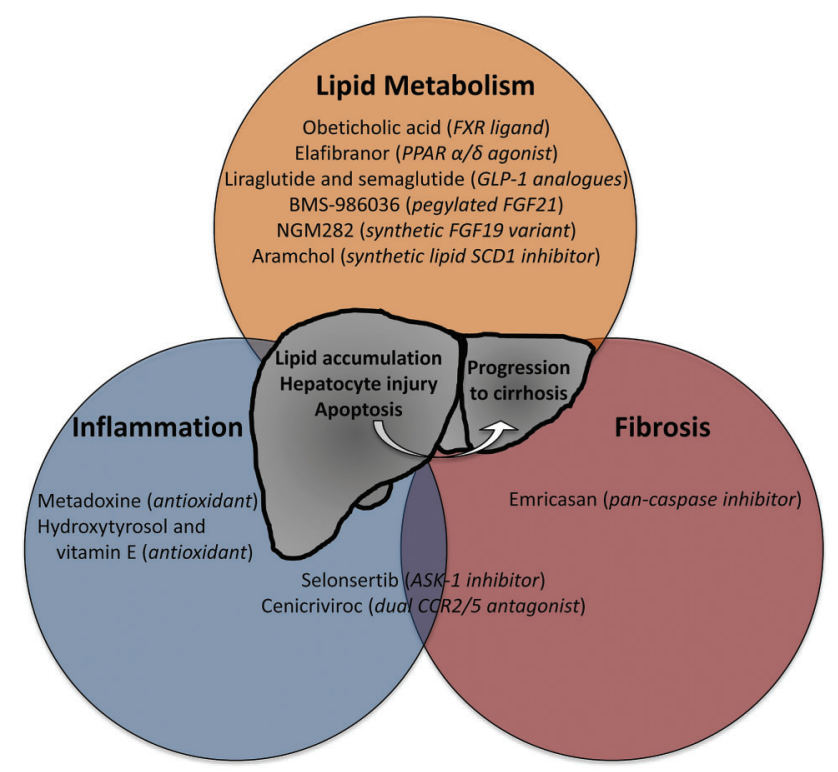

Fig. 1. Key targets for drugs in phase 2 and phase 3 clinical trials.

include farnesoid $X$ nuclear receptor (FXR) agonists, fibroblast growth factor (FGF) variants and peroxisome proliferatoractivated receptor (PPAR) agonists.

FXR is a bile acid receptor ${ }^{9}$ that regulates lipid and glucose metabolism. ${ }^{10}$ FXR activation leads to reduction in serum and hepatic triglyceride levels. ${ }^{11}$ Obeticholic acid is a semi-synthetic FXR agonist currently in a phase 3 trial (national clinical trial number (NCT)02548351).

FGF19 and FGF21 are circulating proteins that bind to FGF receptors (primarily FGFR4 and FGFR1c, respectively) with the cofactor $\beta$-Klotho. ${ }^{12,13}$ In animal models, FGF19 and FGF21 improve lipid profiles (decreasing triglycerides, low-density lipoprotein (LDL) and total cholesterol, and increasing highdensity lipoprotein (HDL)), improve glucose control, and lead to weight loss. ${ }^{14-16}$ In a recent study on animal models, FGF15 (the murine FGF19) fused with apolipoprotein reduced hepatic lipid and bile acid accumulation and improved survival. ${ }^{17}$ Similar results were seen in two phase 1 studies of FGF21 analogues in human subjects. ${ }^{18,19}$ BMS-986036, a pegylated FGF21, and NGM282, a synthetic FGF19 variant, are being studied in phase 2 trials (NCT02443116; NCT02443116).

PPAR- $\alpha, \delta$ and $\gamma$ are transcription factors involved in lipid metabolism. PPAR- $\alpha$ is implicated in fatty acid catabolism. PPAR- $\alpha$ activation increases lipolysis cellular lipid uptake. ${ }^{20}$ Treatment with fibrates, which are PPAR- $\alpha$ ligands, reduces circulating triglycerides and increased HDL levels. ${ }^{21}$ In animal models, PPAR- $\delta$ activation leads to fatty acid consumption in skeletal muscle and adipose tissue. ${ }^{22}$ PPAR- $\gamma$ may reduce hepatic steatosis by shifting fat deposition to adipose tissue. ${ }^{23}$ Rosiglitazone, a PPAR- $\gamma$ agonist, has been shown to reduce liver fat content and laboratory markers of hepatocellular injury, but was also shown to increase cardiovascular risks. ${ }^{24,25}$ Elafibrinor is a PPAR- $\alpha / \delta$ agonist currently in a phase 3 trial (NCT02704403).

Apoptosis signal-regulating kinase 1 , also known as mitogenactivated protein kinase 5 , mediates pathways leading to apoptosis, fibrosis, lipogenesis and the release of inflammatory cytokines. ${ }^{26,27}$ It is activated by angiotensin II receptor type I. Olmesartan, an angiotensin receptor blocker, reduces hepatic steatosis and NASH in animal models. ${ }^{28-30}$ In small trials on patients with cirrhosis, olmesartan reduced serum markers of inflammation. ${ }^{31,32}$ Selonsertib is an apoptosis signal-regulating kinase 1 inhibitor in phase 3 trials for NASH (NCT03053050 and NCT03053063).

As hyperglycemia independently up-regulates fat synthesis via activation of the carbohydrate response element-binding protein transcription factor, ${ }^{33}$ drugs that reduce blood glucose are also being investigated as potential therapies for NASH. Glucagon-like peptide 1 (GLP-1) receptor agonists, also known as an incretin mimetics, may reduce NASH via weight loss and reduction of blood glucose. GLP-1 agonists may influence lipid metabolisms by direct mechanisms as well. ${ }^{34}$ Meta-analysis of patients with diabetes suggests GLP-1 agonists reduce steatosis, inflammation and fibrosis on histology as well as serum markers of inflammation. ${ }^{35}$ Liraglutide is a longacting GLP-1 receptor agonist approved for the treatment of diabetes and is in a phase 3 trial for the treatment of NASH (NCT02654665).

C-C motif chemokine receptor (CCR) 2 and CCR5 are chemokine receptors expressed on circulating monocytes as well as on Kupffer cells. Activation of these receptors induces migration of macrophages into the liver. ${ }^{36-38}$ Cenicriviroc (CVC) is a CCR2/5 antagonist in phase 2 and phase 3 trials (NCT02217475 and NCT03028740).

Caspases are key mediators of inflammation and apoptosis. ${ }^{39}$ Apoptosis is, in turn, profibrotic. ${ }^{40,41}$ Emricasan (IDN6556 ) is a pan-caspase inhibitor that is being studied in phase 2 trials (NCT02686762 and NCT02960204).

\section{Expedited FDA approval process}

The natural history of NASH is typically characterized by a time period of years to decades between the onset of fatty liver to the development of liver cirrhosis and its complications, including liver-related mortality. Regulatory endpoints focused on clinical outcomes such as progression to liver cirrhosis, liver failure, hepatocellular carcinoma, need for liver transplantation, and liver-related death are not feasible within a registration program. Based on evidence confirming the association of surrogate histologic and clinical endpoints and clinical outcomes, the FDA has established regulatory pathways which incorporate non-invasive, clinical and histologic endpoints for phase 2 and 3 clinical development, with the expectation for postmarketing clinical outcome evaluation in phase 4 studies. ${ }^{42}$

Advances in the development of serum biomarkers, imaging and elastography have permitted their use as viable trial endpoints in early human studies, which inform the design of phase 2 trials using a primary endpoint of improvement of $\geq 2$ points in the NAFLD activity score (NAS) including improvement in lobular inflammation or hepatocellular ballooning with no worsening in fibrosis. Phase 3 trials are focused on a primary endpoint of either NASH resolution without worsening of liver fibrosis, or liver fibrosis regression of at minimum one stage without worsening of NASH activity. Phase 4 studies are focused on long-term assessment of clinical outcomes (e.g., all-cause mortality, liver transplant, hepatic decompensation events), progression to cirrhosis, or an increase in model for end-stage liver disease score from $<12$ to $\geq 15.43$ The development of surrogate biomarkers that are "reasonably likely to predict a drug's intended clinical benefit" and are independent of liver histology remains of great interest to clinicians, researchers, and the FDA. ${ }^{44}$ 


\section{Summary of drugs in phase 2 and 3 trials}

We identified eight active phase 3 studies (Table 1 ) and 23 active phase 2 studies (Table 2). Trials of nutritional supplements, such as "Synbiotics Supplement" (NCT02530138), curcumin (NCT02908152), resveratrol (NCT02216552), caffeine and chlorogenic acid (NCT02929901), and CPAP (NCT01849081), were not included.

\section{Drugs in phase 3 trials}

Herein, we report key agents currently under evaluation within the eight ongoing or proposed phase 3 trials registered on ClinicalTrials.gov for the treatment of NASH (Table 1). One study of hydroxytyrosol and vitamin $\mathrm{E}$ for the treatment of children with NASH (NCT02842567) is not reviewed due to its focus on pediatric patients.

Obeticholic acid (OCA): OCA (Intercept Pharmaceuticals, New York, NY, USA) is a farnesoid $X$ nuclear receptor (FXR) ligand that is currently being evaluated in the phase 3 study REGENERATE (NCT02548351) for the treatment of NASH. OCA was granted accelerated approval by the FDA in May 2016 for the treatment of primary biliary cholangitis (PBC) in combination with ursodeoxycholic acid in adults with an inadequate response or intolerance to ursodeoxycholic acid and marketed under the brand name Ocaliva. ${ }^{45}$

In a small phase 2 trial, treatment with OCA was shown to increase insulin sensitivity and reduce alanine aminotransferase levels and serum markers of fibrosis in patients with diabetes and NAFLD. ${ }^{46}$ Subsequently, OCA was studied in the phase 2b FLINT trial (NCT01265498), where 283 patients with non-cirrhotic NASH were randomized 1:1 to receive OCA $25 \mathrm{mg}$ or placebo for 72 weeks (Table 3 ) ${ }^{47}$ The primary outcome was improvement in NAS by $\geq 2$ points without worsening of fibrosis. In both the planned interim analysis and the end of treatment cohorts, OCA demonstrated superiority over placebo in meeting the primary endpoint at 72 weeks on an intention-totreat basis ( $45 \%$ vs. $21 \%, p=0.0002)$, and in addition demonstrated improvement in liver fibrosis (35\% vs. $19 \%, p=0.004)$. Although well-tolerated, a trend for a small increase in LDL and decrease in HDL was identified, but was reversible with HMGCoA reductase inhibitor therapy, resolved spontaneously upon withdrawal of OCA, and was not associated with any difference in cardiovascular events. ${ }^{48}$ Of note, however, are safety concerns, including liver injury, liver decompensation, liver failure and death, that have been reported for a small number of patients with moderate or severe hepatic impairment (ChildPugh $\mathrm{B} / \mathrm{C}$ ) taking OCA for the treatment of PBC. ${ }^{49}$ Although these adverse events were specifically seen in patients with advanced cirrhosis, careful attention to the safety profile of OCA is warranted in phase $3 \mathrm{NASH}$ trials in participants with both normal and impaired hepatic function.

Recruiting for the phase 3 REGNERATE trial began in 2015 with a target $n$ of 2000 participants with biopsy-proven, noncirrhotic NASH to be randomized $1: 1: 1$ to OCA $10 \mathrm{mg}$, OCA $25 \mathrm{mg}$ or placebo groups. The two specified coprimary endpoints are liver fibrosis improving one stage without worsening of $\mathrm{NASH}$, and $\mathrm{NASH}$ resolution with no worsening of fibrosis at 18 months. Other primary outcomes include death or liverrelated adverse events at approximately 6 years. The target primary completion date is October 2021.

Elafibranor: Elafibranor (also known as GFT505) is a dual PPAR- $\alpha / \delta$ agonist produced by GENFIT (Loos, France) that is currently undergoing evaluation in the phase 3 RESOLVE-IT trial (NCT02704403). The drug was granted 'Fast Track' designation by the FDA in February 2014 for the treatment of NASH and received clearance by the FDA in November 2016 for evaluation in PBC. .0, $51^{2}$

Elafibranor was tested in the phase $2 \mathrm{~b}$ GOLDEN-505 trial (NCT01694849) which randomized 276 patients with NASH without cirrhosis to elafibranor $80 \mathrm{mg}$, elafibranor $120 \mathrm{mg}$ or placebo groups in a $1: 1: 1$ fashion (Table 3). ${ }^{52}$ The protocoldefined primary outcome was reversal of NASH on histologic NAS scoring with resolution of steatosis, ballooning, or inflammation without progression to bridging fibrosis or cirrhosis (if bridging fibrosis was evident at baseline) at 52 weeks. Although this primary endpoint was not met in the intention-to-treat analysis, a post-hoc analysis based on a modified definition of response (resolution of NASH defined by disappearance of ballooning with disappearance or mild persistence of lobular inflammation and a pathological diagnosis of steatosis with or without mild inflammation and no worsening of fibrosis) did confirm superiority of the $120 \mathrm{mg}$ dose compared to placebo ( $19 \%$ vs. $12 \%, p=0.045)$, with stronger response among participants with baseline moderate or severe NASH (20\% vs. $11 \%, p=0.018)$. Significant reduction in fibrosis was noted in patients who responded to the $120 \mathrm{mg}$ dose based on the modified definition compared to those who did not. Furthermore, elafibranor was well tolerated, without causing weight gain or cardiac events, and both lipid/glucose profiles and markers of systemic inflammation were reduced. However, a mild, reversible increase in serum creatinine was observed.

The phase 3 RESOLVE-IT trial began in March 2016 with the goal of recruiting 2000 patients with biopsy-proven moderate or severe (F2-F3) NASH. Patients will receive either $120 \mathrm{mg}$ of elafibranor or placebo. The primary endpoints include the proportion of patients with resolution of NASH without worsening of fibrosis and a composite long-term outcome (all-cause mortality, cirrhosis and liver-related clinical outcomes) at 72 weeks with estimated follow-up of 4 years. The study is actively recruiting with an estimated primary completion date in December 2021.

Selonsertib: Selonsertib (also known as GS-4997) is an apoptosis signal-regulating kinase 1 inhibitor produced by Gilead (Foster City, CA, USA) that is currently under evaluation in two phase 3 clinical trials (STELLAR-3 [NCT03053050] and STELLAR-4 [NCT03053063]) for the treatment of NASH. It is intended to reduce JNK- and p38 MAPK-mediated hepatic stellate cell activation and cytokine production. ${ }^{27}$

Early human studies revealed that selonsertib reduces inflammation and hepatocyte apoptosis. ${ }^{53}$ Selonsertib was studied in a phase 2, open label, randomized controlled trial (NCT02466516) conducted throughout the USA and Canada. ${ }^{54}$ It enrolled 72 adults with biopsy-proven NASH and randomized them to receive selonsertib $6 \mathrm{mg}$ or $18 \mathrm{mg}$ with or without simtuzumab for 24 weeks (Table 3 ). The study recruited participants with stage F2-F3 fibrosis and NAS $\geq 5$. Selonsertib was determined to be superior to placebo in achieving the primary efficacy endpoint of fibrosis improvement of one stage or greater $(43 \%$ of $18 \mathrm{mg}-, 30 \%$ of $6 \mathrm{mg}$ - and $20 \%$ of placebo-treated), as well as fibrosis improvement without worsening $\mathrm{NASH}(37 \%$ of $18 \mathrm{mg}-, 30 \%$ of $6 \mathrm{mg}-$ and $20 \%$ of placebo-treated) and progression to cirrhosis ( $3 \%$ of $18 \mathrm{mg}-, 7 \%$ of $6 \mathrm{mg}$ - and $20 \%$ of placebo-treated). However, there was no difference in achieving a decrease in NAS of at minimum two points $(23 \%$ of $18 \mathrm{mg}-$, $19 \%$ of $6 \mathrm{mg}$ - and $20 \%$ of placebo-treated) or NASH resolution ( $0 \%$ of $18 \mathrm{mg}-, 4 \%$ of $6 \mathrm{mg}$ - and $0 \%$ of placebo-treated). 


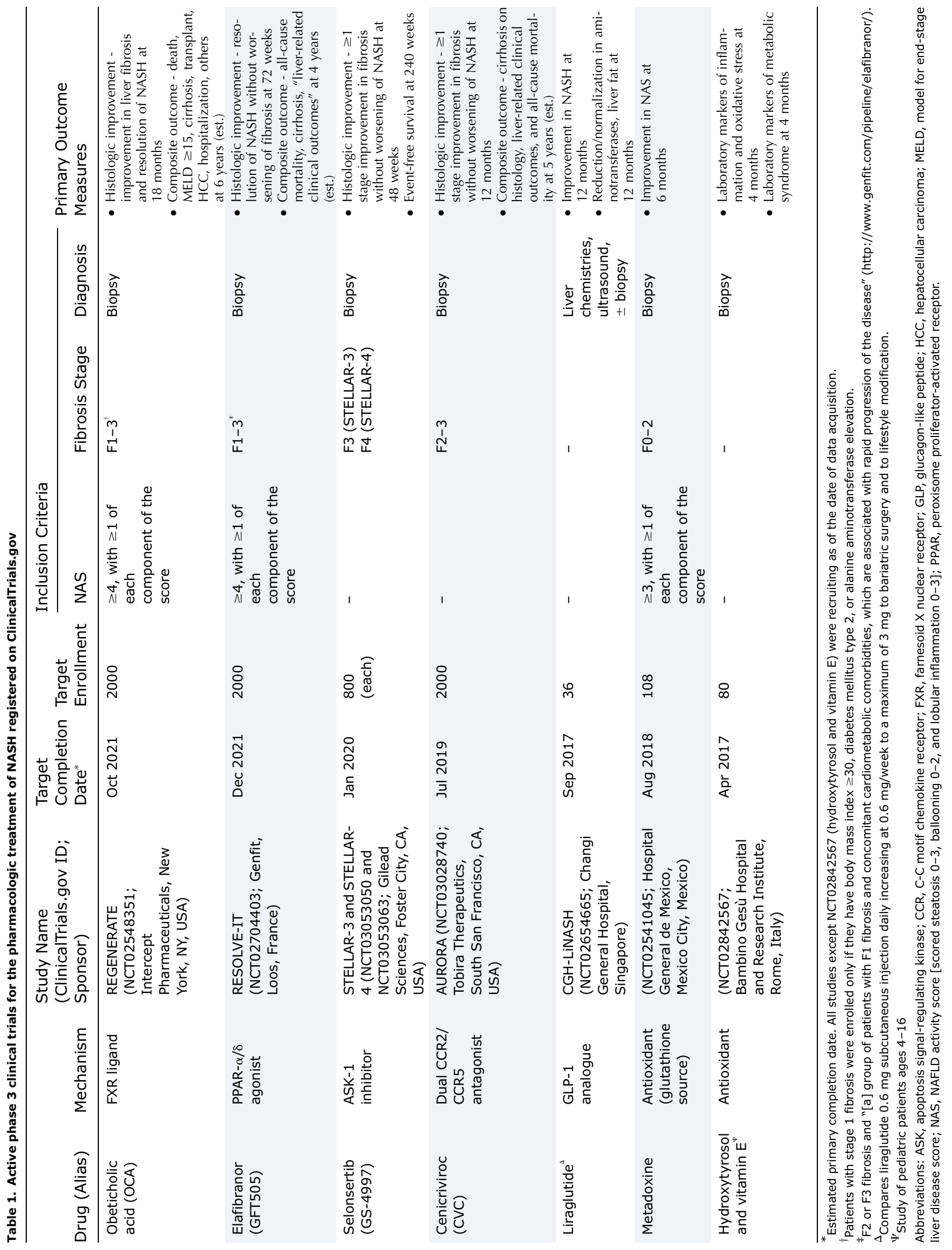




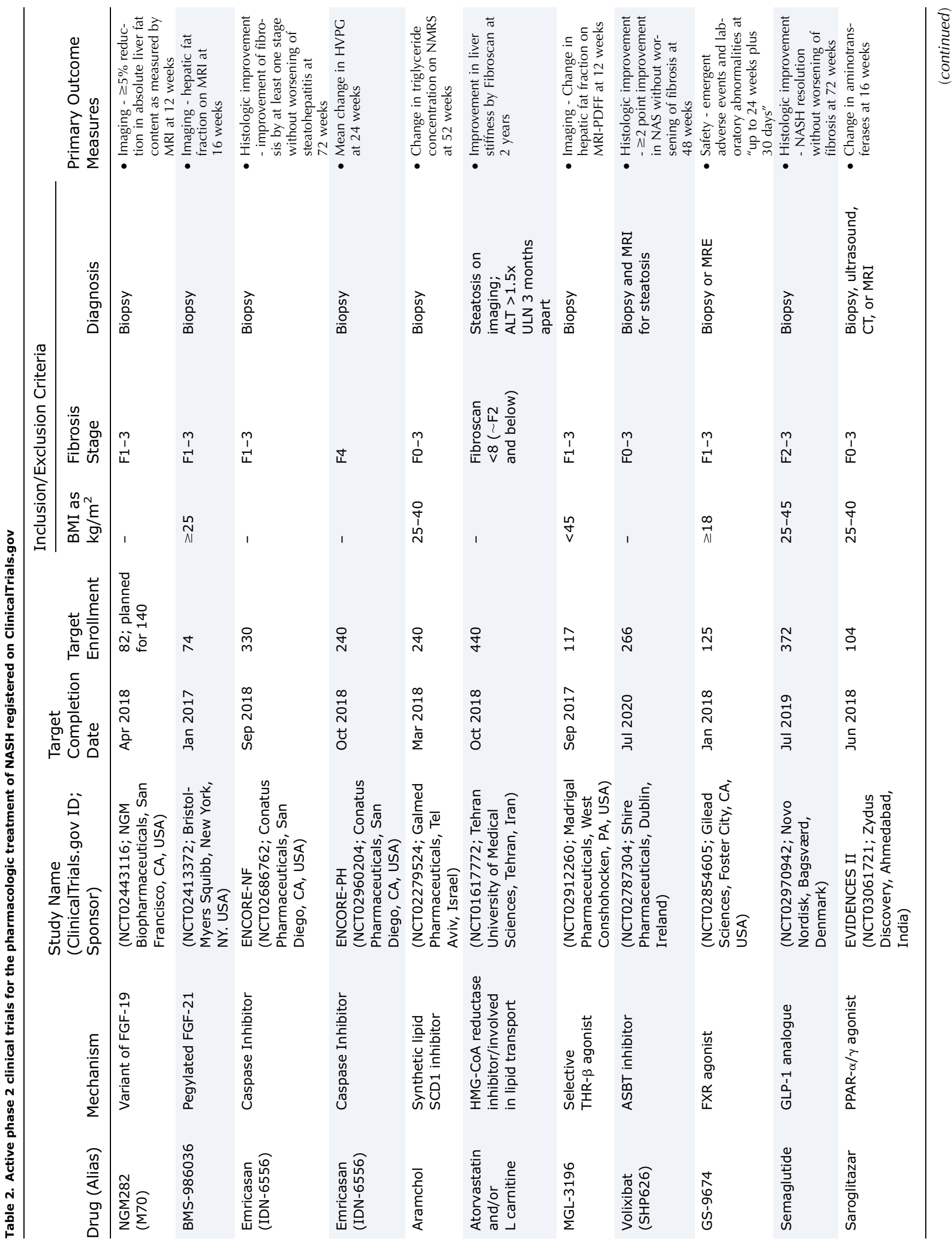




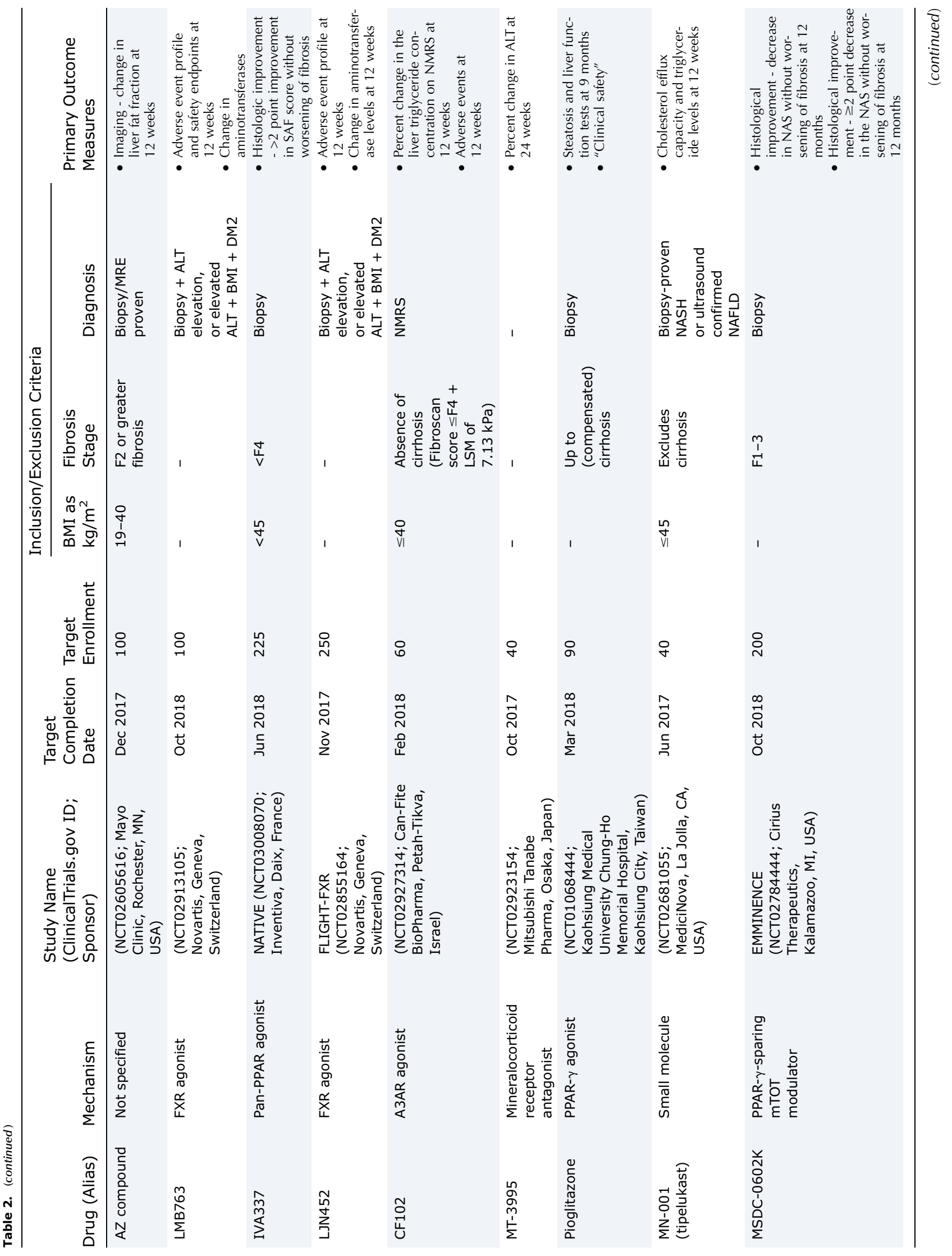




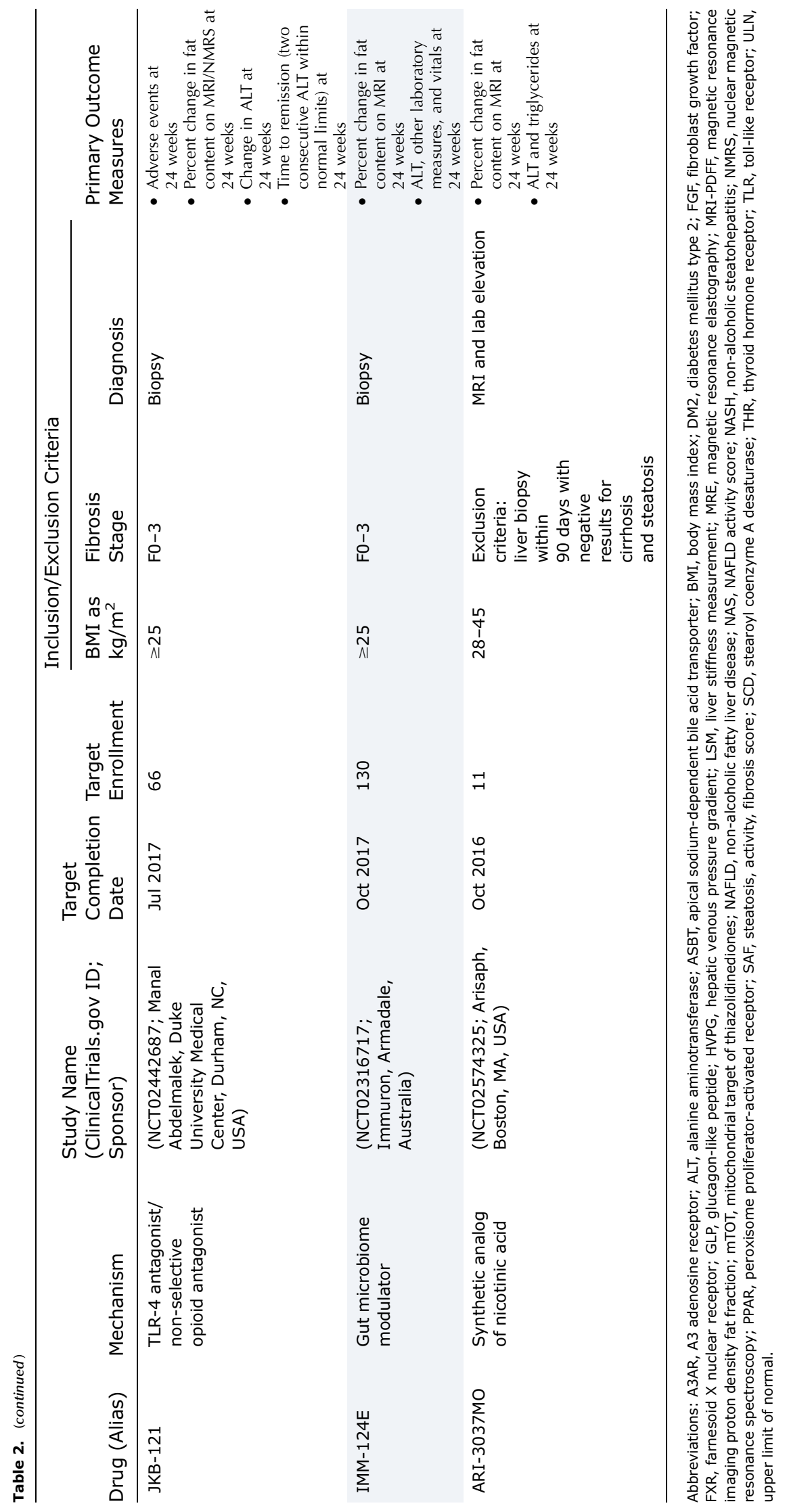


Connolly J.J. et al: Future pharmacotherapy for NASH

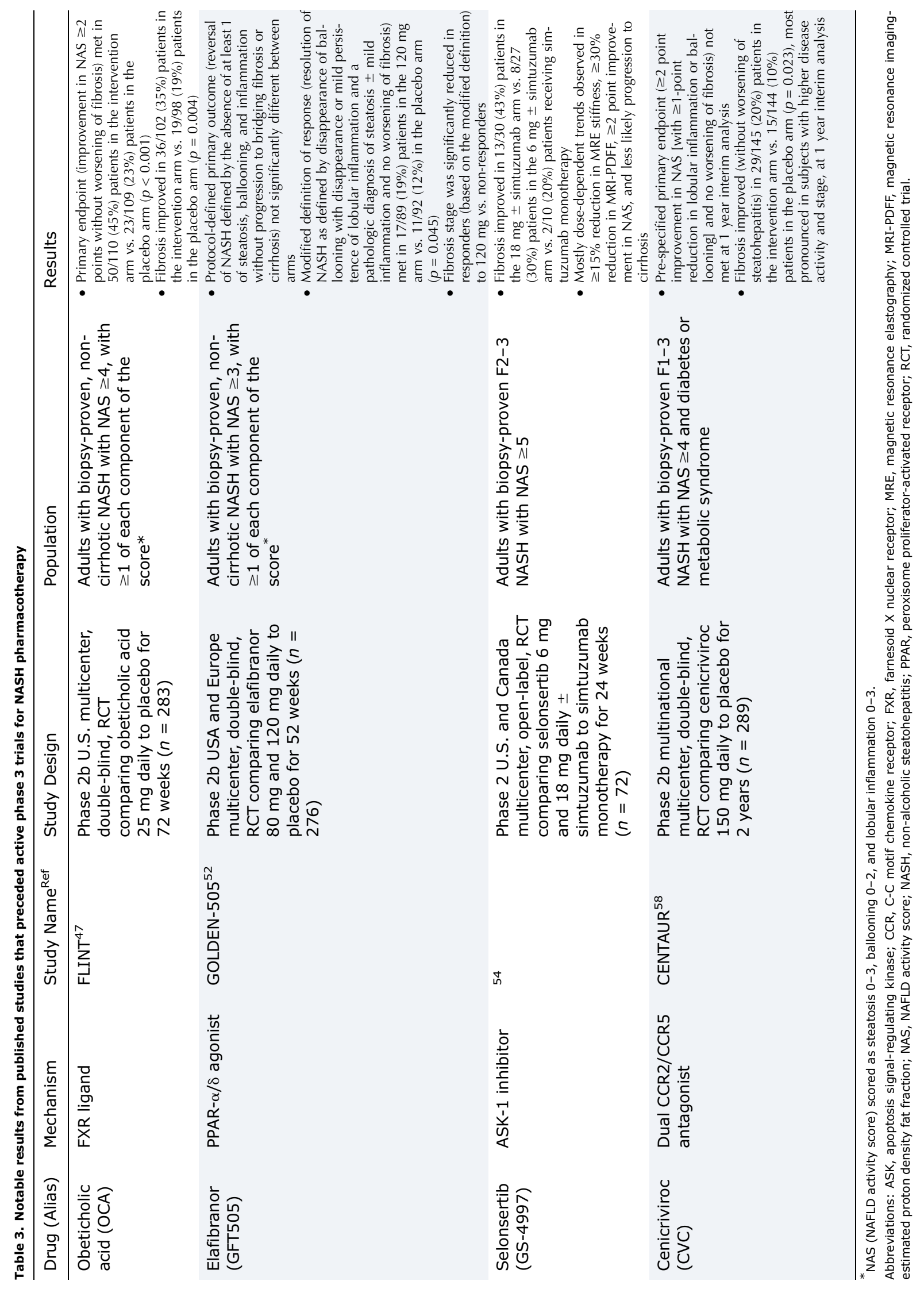


Selonsertib was well-tolerated overall, although key adverse effects included fatigue, headache and nausea.

STELLAR-3 is a multinational phase 3 randomized, placebocontrolled, double-blind clinical trial designed to assess the safety and efficacy of selonsertib $6 \mathrm{mg}$ and $18 \mathrm{mg}$ in patients with stage F3 fibrosis. STELLAR-4 is a phase 3 randomized, placebo-controlled, double-blind clinical trial which evaluates the safety and efficacy of selonsertib in patients with compensated cirrhosis. The primary outcomes for both protocols are liver fibrosis regression $\geq 1$ stage at 48 weeks, and event-free survival at 240 weeks. They are currently recruiting with a target enrollment of 800 participants, each with an estimated completion date of January 2020.

CVC: CVC is an oral, dual CCR2/CCR5 inhibitor owned by Tobira Therapeutics (San Francisco, CA, USA). In animal fibrosis models it is demonstrated to have anti-inflammatory and antifibrotic properties. ${ }^{55-57} \mathrm{CVC}$ is being evaluated in phase 2 and phase 3 trials.

CENTAUR (NCT02217475), a phase 2b study of CVC in 289 patients with $\mathrm{F} 1-\mathrm{F} 3$ fibrosis and one or more of diabetes, body mass index above $25 \mathrm{~kg} / \mathrm{m}^{2}$ with features of metabolic syndrome, and bridging fibrosis (or definite $\mathrm{NASH}$ ), is fully enrolled and ongoing (Table 3 ). The 1 year primary analysis showed that in the intention-to-treat population, the primary endpoint, $a \geq 2$-point improvement in NAS (with $\geq 1$-point reduction in lobular inflammation or hepatocyte ballooning) and no worsening of fibrosis, was not met. ${ }^{58}$ However, twice as many CVC-treated subjects (29/145 [20\%]) compared to placebo $(15 / 144[10 \%])$ attained $\geq 1$ stage improvement in fibrosis without worsening of steatohepatitis $(p=0.023)$. A subgroup analysis of this secondary endpoint identified predictors of response, including NAS $\geq 5$, hepatocellular ballooning grade $\geq 2$, and fibrosis stages F2-F3 ( $p=0.049)$.

AURORA (NCT03028740) is a phase 3 trial of CVC, targeting patients with F2-F3 fibrosis and having an anticipated enrollment of 2000. Primary outcome measures include: 1) histologic improvement of at least one stage in fibrosis without worsening of NASH at 12 months; and 2) a composite endpoint of histologic progression to cirrhosis, liver-related clinical outcomes, and all-cause mortality. The estimated primary completion date is July 2019.

Liraglutide: Liraglutide is a glucagon-like peptide (GLP)-1 analogue approved by the FDA for the treatment of type II diabetes and is marketed under the brand name Victoza. Liraglutide recently demonstrated encouraging results for patients with NASH in several small, randomized trials, 59,60 including the phase 2 LEAN study sponsored by its manufacturer, Novo Nordisk (Bagsværd, Denmark). In this doubleblind, multicenter trial, 52 participants with biopsy-proven NASH (stage FO-F4 fibrosis) were randomized to receive liraglutide or placebo for 48 weeks. The primary outcome was resolution of definitive NASH without worsening of fibrosis from baseline to end of treatment. In the modified intentionto-treat analysis, nine of $23(39 \%)$ patients treated with liraglutide with pre- and posttreatment biopsies had resolution of NASH versus two of $22(9 \%)$ such patients in the placebo group. Patients receiving liraglutide experienced an improvement in weight and hemoglobin A1c, although no significant change in total NAS or fibrosis stage was observed.

Although liraglutide will not be further evaluated in phase 3 development, Novo Nordisk has initiated a phase $2 \mathrm{~b}$ trial (NCT02970942) evaluating another GLP-1 analogue, semaglutide (Table 2) versus placebo in 372 participants with stage F2-F3 fibrosis and NAS $\geq 4$ with a score of at least 1 for each of the components (steatosis, ballooning, and lobular inflammation). The primary outcome is NASH resolution without worsening of fibrosis, and the estimated primary completion date for this protocol is 2019.

Metadoxine: Metadoxine (pyridoxine-L-2-pyrrolidone-5carboxylate) is an antioxidant that is proposed to be a source of glutathione, capable of inhibiting adipocyte differentiation, limiting hepatic lipid accumulation, and exerting antifibrotic properties. $^{61-63}$ It has largely been studied in patients with alcoholic steatosis ${ }^{64}$ and alcoholic hepatitis, ${ }^{65,66}$ and may have a role in managing acute alcohol intoxication, ${ }^{67}$ alcohol dependence, ${ }^{68}$ and attention-deficit/hyperactivity disorder. ${ }^{69}$

In the only significant randomized controlled trial in patients with $\mathrm{NASH}$, metadoxine was compared to placebo for 16 weeks in 134 participants with stage F0-F3 fibrosis. ${ }^{70}$ Although it did not improve liver histology or serum aminotransferases compared to placebo, it did improve steatosis assessed by ultrasound. This improvement in steatosis is consistent with prior results in patients with alcoholic liver disease. ${ }^{64} \mathrm{~A}$ proposed phase 3 trial in Mexico (NCT02541045) of metadoxine in overweight and obese patients with stage F0-F2 fibrosis is currently seeking to enroll 108 patients. The primary outcome is improvement in NAS score at six months and the trial has an estimated completion date of August 2018.

\section{Drugs in phase 2 trials}

Approximately two-dozen phase 2 clinical trials are currently actively looking at novel pharmacotherapies for adults with NASH (Table 2). Of these, only the following three have publicly published results: CVC, as mentioned above in the CENTAUR trial; NGM282 (NGM Biopharmaceuticals, Inc, South San Francisco, CA, USA); and BMS-986036 (BristolMyers Squibb, Princeton, NJ, USA).

NGM282: NGM282 (formerly known as M70) is a nontumorigenic, engineered variant of fibroblast growth factor (FGF)-19 that acts through FGF receptors $1 \mathrm{c}$ and 4 to reduce steatosis and lipotoxicity. It was studied in a randomized, double-blind, placebo controlled phase 2 trial (NCT02443116) in 82 patients with biopsy-proven non-cirrhotic NASH with $\geq 8 \%$ absolute liver fat content by MRI proton density fat fraction (MRI-PDFF). The primary outcome was $a \geq 5 \%$ reduction in absolute liver fat content as measured by MRI-PDFF after 12 weeks of treatment. The results were presented at The International Liver Congress 2017 and demonstrated that $79 \%$ of NMG282-treated subjects met the primary outcome compared with $7 \%$ in the placebo group. ${ }^{71}$ Other findings included lower alanine aminotransferase values and lower enhanced liver fibrosis scores ${ }^{72}$ for the treatment groups, but higher LDL levels were also observed. ${ }^{73}$ Similar results were seen in a phase 2 trial in patients with PBC. ${ }^{74}$ No information is available regarding design of potential phase $2 \mathrm{~b}$ or 3 trials.

BMS-986036: BMS-986036 is a pegylated analogue of FGF-21 that has been shown to decrease hepatic steatosis, NAS and fibrosis in a mouse model of $\mathrm{NASH}^{75}$ and to have beneficial effects on insulin sensitivity, lipids and fibrotic markers in obese diabetic patients with a high prevalence of NAFLD. ${ }^{76,77}$ The results of a phase 2 randomized, doubleblind, placebo controlled trial (NCT02413372) involving 74 participants with a body mass index $\geq 25 \mathrm{~kg} / \mathrm{m}^{2}$, biopsyproven non-cirrhotic NASH, and $\geq 10 \%$ absolute liver fat content by MRI-PDFF were presented at The International Liver Congress 2017. ${ }^{78}$ The primary outcome, a change in percent hepatic fat fraction by MRI-PDFF after 16 weeks of 
treatment, was shown to be statistically significant compared to placebo. Other pertinent outcomes were improvements in adiponectin levels, triglycerides, LDL, HDL, aminotransferases, serum Pro-C3, and liver stiffness measured by magnetic resonance elastography. Bristol-Myers Squibb has suggested further investment in BMS-986036 for the treatment of NASH though no phase $2 b$ or 3 trials have yet been proposed. ${ }^{79}$

\section{Looking ahead}

With the recent advent of highly effective oral direct-acting antiviral agents for the treatment of hepatitis $C$ virus, the prevalence of hepatitis $C$ and its associated burden of liver cirrhosis and its complications is expected to decline. Concurrently, NASH is rapidly emerging as the most common cause of liver cirrhosis and liver failure requiring liver transplantation. In context of a limited treatment paradigm focused on medical weight loss without a single FDA-approved pharmacotherapy for $\mathrm{NASH}$, there exists significant interest in novel agents that may meaningfully alter the natural history of the disease. Several agents are currently in phase 3 clinical trials, and are expected to reach completion within the next 2-4 years. The regulatory framework for drug development remains focused on two pathways to approval, including: 1) NASH resolution without worsening of liver fibrosis; and 2) histologic regression of liver fibrosis of at least one stage without worsening NASH.

Although key clinical development programs have largely evaluated single agents for NASH therapy, future treatment paradigms may involve combination regimens which incorporate two or more complementary mechanisms of action that target metabolic, inflammation, and/or fibrosis factors and/or pathways to optimize efficacy in NASH resolution and liver fibrosis regression. Due to the established association between $\mathrm{NASH}$ and both cardiovascular disease and malignancy, careful attention to off-target adverse effects will be essential. Ongoing scientific advances in the pathogenesis of NASH and identification of novel agents addressing key targets of disease activity will be necessary to further expand our capacity to both treat NASH and reduce long-term clinical outcomes, including cirrhosis, liver failure and liver cancer.

\section{Conflict of interest}

JKL reports research contracts from Allergan, Conatus, Genfit, Gilead, Intercept, and Prometheus. The others have no conflict of interests related to this publication.

\section{Author contributions}

Drafting of the manuscript (JJC, $\mathrm{KO}, \mathrm{JKL})$, contributing to the conception and design of the study (JJC, KO, JKL), contributing to critical revisions of the manuscript (JKL).

\section{References}

[1] Chalasani N, Younossi Z, Lavine JE, Diehl AM, Brunt EM, Cusi K, et al. The diagnosis and management of non-alcoholic fatty liver disease: practice Guideline by the American Association for the Study of Liver Diseases, American College of Gastroenterology, and the American Gastroenterological Association. Hepatology 2012;55:2005-2023. doi: 10.1002/hep.25762.

[2] Younossi ZM, Koenig AB, Abdelatif D, Fazel Y, Henry L, Wymer M. Global epidemiology of nonalcoholic fatty liver disease-Meta-analytic assessment of prevalence, incidence, and outcomes. Hepatology 2016;64:73-84. doi: 10.1002/hep.28431.
[3] Younossi ZM, Blissett D, Blissett R, Henry L, Stepanova M, Younossi Y, et al. The economic and clinical burden of nonalcoholic fatty liver disease in the United States and Europe. Hepatology 2016;64:1577-1586. doi: 10.1002/hep.28785.

[4] Wong RJ, Aguilar M, Cheung R, Perumpail RB, Harrison SA, Younossi ZM, etal. Nonalcoholic steatohepatitis is the second leading etiology of liver disease among adults awaiting liver transplantation in the United States. Gastroenterology 2015;148:547-555. doi: 10.1053/j.gastro.2014.11.039.

[5] Goldberg D, Ditah IC, Saeian K, Lalehzari M, Aronsohn A, Gorospe EC, et al. Changes in the prevalence of hepatitis $C$ virus infection, nonalcoholic steatohepatitis, and alcoholic liver disease among patients with cirrhosis or liver failure on the waitlist for liver transplantation. Gastroenterology 2017;152: 1090-1099.e1. doi: 10.1053/j.gastro.2017.01.003.

[6] Said A, Akhter A. Meta-analysis of randomized controlled trials of pharmacologic agents in non-alcoholic steatohepatitis. Ann Hepatol 2017;16:538547. doi: 10.5604/01.3001.0010.0284.

[7] Lombardi R, Onali S, Thorburn D, Davidson BR, Gurusamy KS, Tsochatzis E. Pharmacological interventions for non-alcohol related fatty liver disease (NAFLD): an attempted network meta-analysis. Cochrane Database Syst Rev 2017;3:CD011640. doi: 10.1002/14651858.CD011640. pub 2.

[8] Singh S, Khera R, Allen AM, Murad MH, Loomba R. Comparative effectiveness of pharmacological interventions for nonalcoholic steatohepatitis: A systematic review and network meta-analysis. Hepatology 2015;62:1417-1432. doi: 10.1002/hep.27999.

[9] Parks DJ, Blanchard SG, Bledsoe RK, Chandra G, Consler TG, Kliewer SA, et al. Bile acids: natural ligands for an orphan nuclear receptor. Science 1999;284: 1365-1368.

[10] Cariou B, van Harmelen K, Duran-Sandoval D, van Dijk TH, Grefhorst A, Abdelkarim $M$, et al. The farnesoid $X$ receptor modulates adiposity and peripheral insulin sensitivity in mice. J Biol Chem 2006;281:11039-11049. doi: $10.1074 /$ jbc.M510258200.

[11] Watanabe M, Houten SM, Wang L, Moschetta A, Mangelsdorf DJ, Heyman RA, et al. Bile acids lower triglyceride levels via a pathway involving $F X R$, SHP, and SREBP-1C. J Clin Invest 2004;113:1408-1418. doi: 10.1172/JCI21025.

[12] Gimeno RE, Moller DE. FGF21-based pharmacotherapy-potential utility for metabolic disorders. Trends Endocrinol Metab 2014;25:303-311. doi: 10. 1016/j.tem.2014.03.001.

[13] Cicione C, Degirolamo C, Moschetta A. Emerging role of fibroblast growth factors $15 / 19$ and 21 as metabolic integrators in the liver. Hepatology 2012;56:2404-2411. doi: 10.1002/hep.25929.

[14] Kharitonenkov A, Wroblewski VJ, Koester A, Chen YF, Clutinger CK, Tigno XT, et al. The metabolic state of diabetic monkeys is regulated by fibroblast growth factor-21. Endocrinology 2007;148:774-781. doi: 10.1210/en.2006-1168.

[15] Bhatnagar S, Damron HA, Hillgartner FB. Fibroblast growth factor-19, a novel factor that inhibits hepatic fatty acid synthesis. J Biol Chem 2009; 284:10023-10033. doi: 10.1074/jbc.M808818200.

[16] Fu L, John LM, Adams SH, Yu XX, Tomlinson E, Renz M, et al. Fibroblast growth factor 19 increases metabolic rate and reverses dietary and leptindeficient diabetes. Endocrinology 2004;145:2594-2603. doi: 10.1210/en. 2003-1671.

[17] Alvarez-Sola G, Uriarte I, Latasa MU, Fernandez-Barrena MG, Urtasun R, Elizalde $M$, et al. Fibroblast growth factor 15/19 (FGF15/19) protects from diet-induced hepatic steatosis: development of an FGF19-based chimeric molecule to promote fatty liver regeneration. Gut 2017;66:1818-1828. doi: $10.1136 /$ gutjinl-2016-312975.

[18] Gaich G, Chien JY, Fu H, Glass LC, Deeg MA, Holland WL, et al. The effects of LY2405319, an FGF21 analog, in obese human subjects with type 2 diabetes. Cell Metab 2013;18:333-340. doi: 10.1016/j.cmet.2013.08.005.

[19] Talukdar S, Zhou Y, Li D, Rossulek M, Dong J, Somayaji V, et al. A long-acting FGF21 molecule, PF-05231023, decreases body weight and improves lipid profile in non-human primates and type 2 diabetic subjects. Cell Metab 2016;23:427-440. doi: 10.1016/j.cmet.2016.02.001.

[20] Ferri N, Corsini A, Sirtori C, Ruscica M. PPAR- $\alpha$ agonists are still on the rise: an update on clinical and experimental findings. Expert Opin Investig Drugs 2017;26:593-602. doi: 10.1080/13543784.2017.1312339.

[21] Staels B, Dallongeville J, Auwerx J, Schoonjans K, Leitersdorf E, Fruchart JC. Mechanism of action of fibrates on lipid and lipoprotein metabolism. Circulation 1998;98:2088-2093. doi: 10.1161/01.CIR.98.19.2088.

[22] Luquet S, Gaudel C, Holst D, Lopez-Soriano J, Jehl-Pietri C, Fredenrich A, et al. Roles of PPAR delta in lipid absorption and metabolism: a new target for the treatment of type 2 diabetes. Biochim Biophys Acta 2005;1740: 313-317. doi: 10.1016/j.bbadis.2004.11.011.

[23] Giby VG, Ajith TA. Role of adipokines and peroxisome proliferator-activated receptors in nonalcoholic fatty liver disease. World J Hepatol 2014;6:570579. doi: 10.4254/wjh.v6.i8.570.

[24] Neuschwander-Tetri BA, Brunt EM, Wehmeier KR, Sponseller CA, Hampton K, Bacon $B R$. Interim results of a pilot study demonstrating the early effects of the PPAR-gamma ligand rosiglitazone on insulin sensitivity, aminotransferases, hepatic steatosis and body weight in patients with non-alcoholic steatohepatitis. J Hepatol 2003;38:434-440. doi: 10.1016/S0168-8278(03)00027-8. 
[25] Nissen SE, Wolski K. Effect of rosiglitazone on the risk of myocardial infarction and death from cardiovascular causes. N Engl J Med 2007;356:24572471. doi: 10.1056/NEJMoa072761.

[26] Hayakawa R, Hayakawa T, Takeda K, Ichijo $\mathrm{H}$. Therapeutic targets in the ASK1-dependent stress signaling pathways. Proc Jpn Acad Ser B Phys Biol Sci 2012;88:434-453. doi: 10.2183/pjab.88.434.

[27] Schuster S, Feldstein AE. NASH: Novel therapeutic strategies targeting ASK1 in NASH. Nat Rev Gastroenterol Hepatol 2017;14:329-330. doi: 10.1038/nrgastro.2017.42

[28] Hirose A, Ono M, Saibara T, Nozaki Y, Masuda K, Yoshioka A, et al. Angiotensin II type 1 receptor blocker inhibits fibrosis in rat nonalcoholic steatohepatitis. Hepatology 2007;45:1375-1381. doi: 10.1002/hep.21638.

[29] Yamamoto E, Dong YF, Kataoka K, Yamashita T, Tokutomi Y, Matsuba S, et al. Olmesartan prevents cardiovascular injury and hepatic steatosis in obesity and diabetes, accompanied by apoptosis signal regulating kinase-1 inhibition. Hypertension 2008;52:573-580. doi: 10.1161/HYPERTENSIONAHA. 108.112292.

[30] Sturzeneker MC, Ioshii SO, Villela Baroncini LA, Précoma DB. Olmesartan severely weakened the development of NASH in an animal model of hypercholesterolemia. Atherosclerosis 2011;216:97-102. doi: 10.1016/j.atherosclerosis.2011.01.047.

[31] Hidaka H, Ohbu M, Matsumoto $Y$, Minamino $T$, Takada J, Takada $Y$, et al. Olmesartan for non-alcoholic steatohepatitis complicated with hypertension: An open-label study. Open Journal of Gastroenterology 2013;3:128-133. doi: 10.4236/ojgas.2013.32021.

[32] Hidaka H, Nakazawa T, Shibuya A, Minamino T, Takada J, Tanaka Y, et al. Effects of 1-year administration of olmesartan on portal pressure and TGFbeta 1 in selected patients with cirrhosis: a randomized controlled trial. J Gastroenterol 2011;46:1316-1323. doi: 10.1007/s00535-011-0449-z.

[33] Ishii S, Iizuka K, Miller BC, Uyeda K. Carbohydrate response element binding protein directly promotes lipogenic enzyme gene transcription. Proc Natl Acad Sci U S A 2004;101:15597-15602. doi: 10.1073/pnas.0405238101.

[34] Petit JM, Vergès B. GLP-1 receptor agonists in NAFLD. Diabetes Metab 2017; 43:2S28-2S33. doi: 10.1016/S1262-3636(17)30070-8.

[35] Dong Y, Lv Q, Li S, Wu Y, Li L, Li J, et al. Efficacy and safety of glucagon-like peptide-1 receptor agonists in non-alcoholic fatty liver disease: A systematic review and meta-analysis. Clin Res Hepatol Gastroenterol 2017;41:284295. doi: 10.1016/j.clinre.2016.11.009.

[36] Gadd VL, Patel PJ, Jose S, Horsfall L, Powell EE, Irvine KM. Altered peripheral blood monocyte phenotype and function in chronic liver disease: implications for hepatic recruitment and systemic inflammation. PLoS One 2016;11: e0157771. doi: 10.1371/journal.pone.0157771.

[37] Seki E, de Minicis S, Inokuchi S, Taura K, Miyai K, van Rooijen N, et al. CCR2 promotes hepatic fibrosis in mice. Hepatology 2009;50:185-197. doi: 10. 1002/hep.22952.

[38] Baeck C, Wehr A, Karlmark KR, Heymann F, Vucur M, Gassler N, et al. Pharmacological inhibition of the chemokine CCL2 (MCP-1) diminishes liver macrophage infiltration and steatohepatitis in chronic hepatic injury. Gut 2012; 61:416-426. doi: 10.1136/gutjnl-2011-300304.

[39] Chang HY, Yang X. Proteases for cell suicide: functions and regulation of caspases. Microbiol Mol Biol Rev 2000;64:821-846. doi: 10.1128/MMBR. 64.4.821-846.2000.

[40] Galle PR, Krammer PH. CD95-induced apoptosis in human liver disease. Semin Liver Dis 1998;18:141-151. doi: 10.1055/s-2007-1007150.

[41] Yoon JH, Gores G]. Death receptor-mediated apoptosis and the liver. J Hepatol 2002;37:400-410. doi: 10.1016/S0168-8278(02)00209-X.

[42] Guidance for industry: Expedited programs for serious conditions-drugs and biologics. Silver Spring: US. Food and Drug Administration 2014. Available from: https://www.fda.gov/downloads/Drugs/GuidanceComplianceRegulatoryInformation/Guidances/UCM358301.pdf.

[43] Omokaro SO. FDA Introductory Remarks. Liver Forum. Amsterdam, The Netherlands 2017.

[44] Sanyal AJ, Miller V. Regulatory science and drug approval for alcoholic and nonalcoholic steatohepatitis. Gastroenterology 2016;150:1723-1727. doi: $10.1053 / j$.gastro.2016.02.044

[45] FDA grants accelerated approval to ocaliva ${ }^{\mathrm{TM}}$ (obeticholic acid) for the treatment of patients with PBC. Intercept Pharmaceuticals, Inc. 2016. Available from: https://globenewswire.com/news-release/2016/05/28/844143/0/en/FDAGrants-Accelerated-Approval-to-Ocaliva-Obeticholic-Acid-for-the-Treatmentof-Patients-with-PBC.html.

[46] Mudaliar S, Henry RR, Sanyal AJ, Morrow L, Marschall HU, Kipnes M, et al. Efficacy and safety of the farnesoid $X$ receptor agonist obeticholic acid in patients with type 2 diabetes and nonalcoholic fatty liver disease. Gastroenterology 2013;145:574-582.e1. doi: 10.1053/j.gastro.2013.05.042.

[47] Neuschwander-Tetri BA, Loomba R, Sanyal AJ, Lavine JE, Van Natta ML, Abdelmalek MF, et al. Farnesoid $\mathrm{X}$ nuclear receptor ligand obeticholic acid for non-cirrhotic, non-alcoholic steatohepatitis (FLINT): a multicentre, randomised, placebo-controlled trial. Lancet 2015;385:956-965. doi: 10.1016/ S0140-6736(14)61933-4.
[48] Hameed B, Terrault N, Gill RM, Loomba R, Chalasani NP, Hoofnagle JH, et al. Clinical and metabolic effects associated with weight loss and obeticholic acid in nonalcoholic steatohepatitis (NASH). Hepatology 2015;62:331A.

[49] FDA Drug Safety Communication: FDA warns about serious liver injury with Ocaliva (obeticholic acid) for rare chronic liver disease. U.S. Food \& Drug Administration 2017. Available from: https://www.fda.gov/Drugs/DrugSafety/ucm576656.htm.

[50] GENFIT: the FDA grants fast track designation to gft505 in NASH. Lille, France 2014. Available from: http://www.genfit.com/wp-content/uploads/ 2014/03/2014.02.17_PR-GENFIT_EN.pdf.

[51] GENFIT: FDA has officially cleared the IND to proceed with Phase II trial and evaluate elafibranor in PBC. Lille, France 2016. Available from: http://www. genfit.com/press-release/genfit-fda-officially-cleared-ind-proceed-phase-iitrial-evaluate-elafibranor-pbc/.

[52] Ratziu V, Harrison SA, Francque S, Bedossa P, Lehert P, Serfaty L, et al. Elafibranor, an agonist of the peroxisome proliferator-activated receptor- $\alpha$ and $-\delta$, induces resolution of nonalcoholic steatohepatitis without fibrosis worsening. Gastroenterology 2016;150:1147-1159.e5. doi: 10.1053/j. gastro.2016.01.038.

[53] Diehl AM, French D, Xu R, Loomba R, Lawitz E, Freilich B, et al. Treatment with selonsertib, an inhibitor of apoptosis signal-regulating kinase 1 , hepatic phospho-p38 expression and markers of hepatocellular apoptosis and necrosis in patients with nonalcoholic steatohepatitis. J Hepatol 2017;66: S51-S52. doi: 10.1016/S0168-8278(17)30366-5.

[54] Loomba R, Lawitz E, Mantry PS, Jayakumar S, Caldwell SH, Arnold H, et al. The ASK1 inhibitor selonsertib in patients with nonalcoholic steatohepatitis: A randomized, phase 2 trial. Hepatology 2017. doi: 10.1002/hep.29514.

[55] Lefebvre E, Moyle G, Reshef R, Richman LP, Thompson M, Hong F, et al. Antifibrotic Effects of the Dual CCR2/CCR5 Antagonist Cenicriviroc in Animal Models of Liver and Kidney Fibrosis. PLoS One 2016;11:e0158156. doi: 10.1371/journal.pone.0158156.

[56] Kruger A], Fuchs BC, Vig P, Lefebvre E, Holmes JA, Masia R, et al. Cenicriviroc (CVC), a dual inhibitor of chemokine receptors (CCR) 2 and 5, decreases hepatic inflammation by altering inflammatory macrophage populations in a mouse model of NASH. Hepatology 2016;64:777A.

[57] Puengel T, Krenkel O, Mossanen J, Longerich T, Lefebvre E, Trautwein C, et al. The dual CCR2/CCR5 antagonist cenicriviroc ameliorates steatohepatitis and fibrosis in vivo by inhibiting the infiltration of inflammatory monocytes into injured liver. J Hepatol 2016;64:S160. doi: 10.1016/S0168-8278(16)01667-6.

[58] Sanyal AJ, Ratziu V, Harrison S, Abdelmalek MF, Aithal GP, Caballeria J, et al. Cenicriviroc placebo for the treatment of non-alcoholic steatohepatitis with liver fibrosis: Results from the Year 1 primary analysis of the Phase 2b CENTAUR study. Hepatology 2016;64:1118A-1119A. doi: 10.1002/hep.28909.

[59] Armstrong MJ, Gaunt P, Aithal GP, Barton D, Hull D, Parker R, et al. Liraglutide safety and efficacy in patients with non-alcoholic steatohepatitis (LEAN): a multicentre, double-blind, randomised, placebo-controlled phase 2 study. Lancet 2016;387:679-690. doi: 10.1016/S0140-6736(15)00803-X.

[60] Eguchi Y, Kitajima Y, Hyogo H, Takahashi H, Kojima M, Ono M, et al. Pilot study of liraglutide effects in non-alcoholic steatohepatitis and non-alcoholic fatty liver disease with glucose intolerance in Japanese patients (LEAN-J). Hepatol Res 2015;45:269-278. doi: 10.1111/hepr.12351.

[61] Yang YM, Kim HE, Ki SH, Kim SG. Metadoxine, an ion-pair of pyridoxine and L-2-pyrrolidone-5-carboxylate, blocks adipocyte differentiation in association with inhibition of the PKA-CREB pathway. Arch Biochem Biophys 2009; 488:91-99. doi: 10.1016/j.abb.2009.07.007.

[62] Muriel P, Deheza R. Fibrosis and glycogen stores depletion induced by prolonged biliary obstruction in the rat are ameliorated by metadoxine. Liver Int 2003;23:262-268. doi: 10.1034/j.1600-0676.2003.00837.x.

[63] Calabrese V, Calderone A, Ragusa N, Rizza V. Effects of Metadoxine on cellular status of glutathione and of enzymatic defence system following acute ethanol intoxication in rats. Drugs Exp Clin Res 1996;22:17-24.

[64] Caballería J, Parés A, Brú C, Mercader J, García Plaza A, Caballería L, et al. Metadoxine accelerates fatty liver recovery in alcoholic patients: results of a randomized double-blind, placebo-control trial. Spanish Group for the Study of Alcoholic Fatty Liver. J Hepatol 1998;28:54-60. doi: 10.1016/S0168-8278 (98)80202-X.

[65] Higuera-de la Tijera F, Servín-Caamaño AI, Serralde-Zúñiga AE, Cruz-Herrera J, Pérez-Torres E, Abdo-Francis JM, et al. Metadoxine improves the three- and six-month survival rates in patients with severe alcoholic hepatitis. World J Gastroenterol 2015;21:4975-4985. doi: 10.3748/wjg.v21.i16.4975.

[66] Higuera-de la Tijera F, Servín-Caamaño AI, Cruz-Herrera J, Serralde-Zúñiga $\mathrm{AE}$, Abdo-Francis JM, Gutiérrez-Reyes $\mathrm{G}$, et al. Treatment with metadoxine and its impact on early mortality in patients with severe alcoholic hepatitis. Ann Hepatol 2014;13:343-352.

[67] Shpilenya LS, Muzychenko AP, Gasbarrini G, Addolorato G. Metadoxine in acute alcohol intoxication: a double-blind, randomized, placebo-controlled study. Alcohol Clin Exp Res 2002;26:340-346. doi: 10.1111/j.1530-0277. 2002.tb02543.x.

[68] Leggio L, Kenna GA, Ferrulli A, Zywiak WH, Caputo F, Swift RM, et al. Preliminary findings on the use of metadoxine for the treatment of alcohol 
dependence and alcoholic liver disease. Hum Psychopharmacol 2011;26: 554-559. doi: 10.1002/hup.1244.

[69] Manor I, Ben-Hayun R, Aharon-Peretz J, Salomy D, Weizman A, Daniely Y, et al. A randomized, double-blind, placebo-controlled, multicenter study evaluating the efficacy, safety, and tolerability of extended-release metadoxine in adults with attention-deficit/hyperactivity disorder. J Clin Psychiatry 2012; 73:1517-1523. doi: 10.4088/JCP.12m07767.

[70] Shenoy KT, Balakumaran LK, Mathew P, Prasad M, Prabhakar B, Sood A, et al. Metadoxine Versus Placebo for the Treatment of Non-alcoholic Steatohepatitis: A Randomized Controlled Trial. J Clin Exp Hepatol 2014;4:94-100. doi: 10.1016/j.jceh.2014.03.041

[71] Harrison SA, Abdelmalek MF, Trotter JF, Paredes AH, Arnold HL, Kugelmas M, et al. NGM282, a novel variant of FGF19, significantly reduces hepatic steatosis and key biomarkers of NASH: results of a Phase 2, multicenter, randomized, double-blinded, placebo controlled trial in biopsy-confirmed NASH patients. J Hepatol 2017;66:S92-S93. doi: 10.1016/S0168-8278(17)30448-8.

[72] Lichtinghagen R, Pietsch D, Bantel H, Manns MP, Brand K, Bahr MJ. The Enhanced Liver Fibrosis (ELF) score: normal values, influence factors and proposed cut-off values. J Hepatol 2013;59:236-242. doi: 10.1016/j.jhep.2013. 03.016.

[73] Luo J, Ko B, Ding X, Rossi S, DePaoli A, Tian H. Serum cholesterol changes associated with NGM282 treatment in obese insulin resistant cynomolgus monkeys are reversed with either a statin or a PCSK9 inhibitor. J Hepato 2017;66:S430. doi: 10.1016/S0168-8278(17)31227-8.
[74] Mayo MJ, Wigg AJ, Roberts SK, Arnold $\mathrm{H}$, Hassanein TI, Leggett BA, et al. NGM282, a novel variant of FGF-19, demonstrates biologic activity in primary biliary cirrhosis patients with an incomplete response to ursodeoxycholic acid: results of a phase 2 multicenter, randomized, double blinded, placebo controlled trial. Hepatology 2015;62:263A-264A.

[75] Krupinski J, Morgan N, Kozhich A, Chiney M, Morin P, Christian R. Effects of BMS-986036 (pegylated fibroblast growth factor 21) on hepatic steatosis and fibrosis in a mouse model of nonalcoholic steatohepatitis. Hepatology 2016;64:749A.

[76] Charles ED, Morrow L, Hompesch M, Luo Y, Wu CK, Christian R. A phase 1 study of BMS-986036 (pegylated FGF21) in healthy obese subjects. Hepatology 2016;64:546A.

[77] Charles ED, Tetri BA, Luo Y, Wu CK, Christian R. A phase 2 study of BMS986036 (pegylated FGF21) in obese adults with type 2 diabetes and a high prevalence of fatty liver. Hepatology 2016:64:17A.

[78] Sanyal A, Charles ED, Neuschwander-Tetri B, Loomba R, Harrison S, Abdelmalek M, et al. BMS-986036 (pegylated FGF21) in patients with non-alcoholic steatohepatitis: a phase 2 study. J Hepatol 2017;66:S89-S90. doi: 10. 1016/S0168-8278(17)30443-9.

[79] Bristol-Myers Squibb's BMS-986036 (Pegylated FGF21) shows consistent improvement in liver fat, liver injury and fibrosis in patients with nonalcoholic steatohepatitis (NASH) in phase 2 trial. Princeton 2017. Available from: https://news.bms.com/press-release/bmy/bristol-myers-squibbs-bms-986036pegylated-fgf21-shows-consistent-improvement-live. 